\title{
Performance Assessment of the World Wide Lightning Location Network (WWLLN), Using the Los Alamos Sferic Array (LASA) as Ground Truth
}

\author{
Abram R. Jacobson AND Robert Holzworth \\ Department of Earth and Space Sciences, University of Washington, Seattle, Washington \\ JEREMIAH HARLiN \\ Space and Remote Sensing Sciences Group, Los Alamos National Laboratory, Los Alamos, New Mexico \\ RICHARD DOWDEN \\ Low Frequency Electromagnetics, Dunedin, New Zealand \\ ERIN LAY \\ Department of Earth and Space Sciences, University of Washington, Seattle, Washington
}

(Manuscript received 11 June 2005, in final form 5 December 2005)

\begin{abstract}
The World Wide Lighting Location Network (WWLLN) locates lightning globally, using sparsely distributed very low frequency (VLF) detection stations. Due to WWLLN's detection at VLF (in this case $\sim 10$ $\mathrm{kHz}$ ), the lightning signals from strong strokes can propagate up to $\sim 10^{4} \mathrm{~km}$ to WWLLN sensors and still be suitable for triggering a station. A systematic evaluation of the performance of WWLLN is undertaken, using a higher-frequency $(0-500 \mathrm{kHz})$ detection array [the Los Alamos Sferic Array (LASA)] as a ground truth during an entire thunderstorm season in a geographically confined case study in Florida. It is found that (a) WWLLN stroke-detection efficiency rises sharply to several percent as the estimated lightning current amplitude surpasses $\sim 30 \mathrm{kA}$; (b) WWLLN spatial accuracy is around $15 \mathrm{~km}$, good enough to resolve convective-storm cells within a larger storm complex; (c) WWLLN is able to detect intracloud and cloudto-ground discharges with comparable efficiency, as long as the current is comparable; (d) WWLLN detects lightning-producing storms with high efficiency in every 3-h epoch; thus, WWLLN can be useful for locating deep convection for weather forecasting on 3-h update cycles; and (e) WWLLN detects a stroke count in each storm that is weakly proportional to the stroke count detected by LASA. Thus, to the extent that lightning rate can serve as a statistical proxy for rainfall, WWLLN may eventually provide rainfall-proxy data to be assimilated in 3 -h forecast update cycles.
\end{abstract}

\section{Introduction}

It is now widely recognized that real-time remote sensing of lightning can assist in identification and monitoring of severe convective weather. Different lightning remote sensing systems have complementary levels of detail, range, and application. The finest lightning detail, but also the most restricted geographical

Corresponding author address: Abram R. Jacobson, Department of Earth and Space Sciences, University of Washington, Box 351310, Seattle, WA 98195-1310.

E-mail: abramj@u.washington.edu range, is provided by very high frequency (VHF) lightning-mapping arrays (LMAs), amongst which the New Mexico Institute of Mining and Technology's LMA has been the best-known system (Rison et al. 1999; Thomas et al. 2000, 2001). Affording less detail, but potentially unlimited geographical range, is space-based optical lightning detection from geostationary orbit (Christian et al. 1989). Toward this end, the OTD and LIS lightning imagers from low-earth-orbit (Boccippio et al. 2000a,b, 1999; Christian et al. 1999a,b) have recently demonstrated that satellite optical imaging can provide a monitor of "total lightning," that is, both cloud-toground (CG) and intracloud (IC) discharges, with high detection efficiency (DE) and global coverage. 
For ground-based monitoring of CG activity, lowfrequency (LF) detection arrays, exemplified by the U.S. National Lightning Detection Network (NLDN), give excellent coverage of almost all continental storms, which account for most global lightning (Cummins et al. 1998). NLDN and similar systems provide not only accurate location $(<1 \mathrm{~km}$ errors) and high DE $(>90 \%)$, but also an estimate of discharge vertical-current amplitude and polarity. NLDN-like systems now operate on parts of most continents. These systems provide operationally useful real-time information for weatherhazard mitigation, forest-fire response, and electric-grid emergency response. NLDN and similar systems have also provided a wealth of scientific information (Carey et al. 2003; Lyons et al. 1998; Orville 1994; Orville et al. 2001) and precise benchmarks against which to calibrate the performance of other systems (Jacobson et al. 2000; Lay et al. 2004; Smith et al. 2002).

The Los Alamos Sferic Array (LASA) is a variation of the NLDN-style low-frequency array. LASA has been implemented (Smith et al. 2002) for specific, geographically delimited campaigns in which it is vital to record and archive the LF waveform from each station participating in a lightning detection/location. LASA has been recently upgraded to allow nearly continuous waveform recording with no "dead time" (Shao et al. 2006). LASA waveform data can be used to identify the lightning process by detailed examination of the waveform, and more importantly, the waveforms for certain IC discharges can be used to retrieve the discharge height above the local ground (Smith et al. 2004). Unlike NLDN, LASA is purely a research facility and is not meant to provide either the geographical coverage, or the up-time reliability, of NLDN.

Providing the least remote sensing detail, but also the greatest range of detection at the lowest cost, is the approach based on detecting vertical-electric-field disturbances in the VLF $(3-30 \mathrm{kHz})$ spectral band. This was begun by the Met Office of the U.K. (Lee 1986a,b) and has more recently provided the basis of other deployed systems including the World Wide Lightning Location Network (WWLLN) (Dowden et al. 2002; Lay et al. 2004; Rodger et al. 2004, 2005).

Long-range (thousands of kilometers) VLF lightning detection can, in principle, provide global coverage, including over remote oceanic regions, for a very modest investment. VLF can do this because its signals are less attenuated than are higher frequencies, such that a VLF array containing on the order of merely 20 stations distributed globally can provide useful coverage of all Tropics and midlatitudes. The penalty is that the wave energy arrives at the sensors via the earth-ionosphere waveguide rather than by line-of-sight ("ground wave"). A VLF system bandwidth is on the order of only $10 \mathrm{kHz}$, and this already is marginal for retrieving the characteristics of the lightning current waveform even in the case of ground-wave propagation from proximate events. The loss of source characteristics is further worsened by the reflections from the ionosphere and the propagation multipath (earth-ionosphere waveguiding.) The ionospheric interaction spectrally distorts and attenuates the received waveform, so that it is no longer straightforward to infer the verticalcurrent magnitude or, for that matter, even the verticalcurrent polarity.

Nonetheless, VLF systems for lightning detection are potentially useful in providing weather data over wide regions, for real-time information in aviation management, for climate studies, and for meteorological forecasting. To perform best, the VLF systems should preferably meet at least three standards:

1) efficient storm detection,

2) proportional stroke detection, and

3) minimal false detections.

The remainder of this article will assess the WWLLN system against these three performance standards.

\section{System description}

LASA has been a reliable lightning-location array over the Florida region. In addition, LASA records and archives digitized waveforms of the vertical-electricfield transients at each station that participates in a lightning event's time-difference-of-arrival (TDOA) location of the lightning discharge. The purpose of LASA is to support various lightning projects of the Department of Energy (DOE) and to provide waveforms to lightning researchers. LASA covers only a small fraction of North America, and in the case of this study, the Florida region. LASA is not intended to supplant the NLDN, which provides (at modest cost) reliable (Cummins et al. 1998) stroke- or flash-level lightning location and current estimates over all of North America, at all times.

The LASA system during 2004 operated in its new mode (Stanley et al. 2004), whereby there are no "dead times" between successive recordings. Each record was $1 \mathrm{~ms}$ in duration. Thus, during a flash containing multiple discharges, LASA can easily record all successive members of the flash, providing that the stroke verticalcurrent amplitude exceeds a few kiloamps, and providing that the location is interior to the array. In practice that means lying within a $400-\mathrm{km}$-radius circle centered on $29^{\circ} \mathrm{N}, 82^{\circ} \mathrm{W}$. [This new array center is displaced $0.5^{\circ} \mathrm{W}$ and $1^{\circ} \mathrm{N}$ relative to the "old" LASA array cen- 


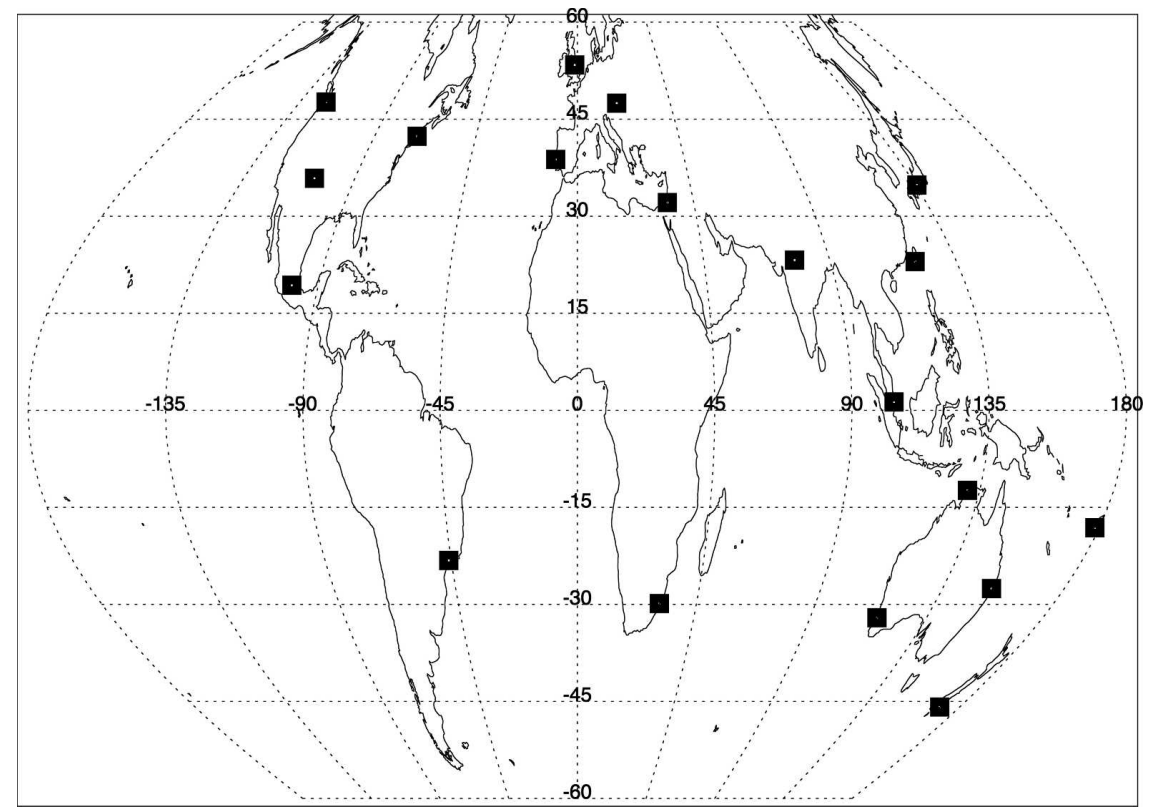

FIG. 1. Locations of the 19 WWLLN stations that were routinely operating during this campaign. The Florida Peninsula is sufficiently far from all stations to require ionosphereearth-waveguide signal propagation to the sensors.

ter, which obtained during 1999-2002 (Jacobson and Heavner 2005).] For records containing more than one lightning pulse, the 2004 upgrade of LASA allows multiple solutions to be tested and, if appropriate, archived. For example, the descending leader prior to a negative CG stroke can create pulses contained within the same 1-ms-duration record that contains the main CG stroke.

LASA detects in-cloud discharges of sufficient amplitude. For the purposes of this paper, we will be ultimately focusing on current amplitudes exceeding 30 $\mathrm{kA}$. In practice, practically all of the LASA-detected IC events of this high a current are "narrow bipolar events" (NBEs; Smith et al. 1999, 2002).

Figure 1 shows the WWLLN station locations during the time of this study (summer 2004). The closest WWLLN stations to Florida were in Boston, Massachusetts; Los Alamos, New Mexico; and Seattle, Washington. All of these are separated from the LASA array center by distances that are large compared to the nominal maximum ground-wave distance $(\sim 600 \mathrm{~km})$, beyond which ionospheric pathing may be expected to interfere with, and ultimately (at larger distances) dominate over the ground wave. We required at least five WWLLN stations in each TDOA location solution. All the WWLLN participating stations in this study were detecting the lightning events via earthionosphere-waveguide propagation paths. The WWLL lightning-location solutions were generated using the upgraded "time of group arrival" (TOGA) algorithm at each WWLLN station (Dowden et al. 2002). The WWLLN array station locations and other system parameters can be found online at http://flash.ess. washington.edu/. During the time of this study, there were a maximum of 19 WWLLN stations worldwide.

The quality of WWLL location solutions is governed, in part, by the root-mean-square (rms) residual between the observed and the modeled arrival times of a signal at participating stations. For this study, we used solutions with rms residuals less than $30 \mu \mathrm{s}$.

Each WWLLN station has a servo-controlled trigger threshold that seeks to keep a constant trigger rate, on the order of five triggers per second. This means that the average intertrigger time will be on the order of $0.2 \mathrm{~s}$. WWLLN stroke detections generally do not occur more than once within a lightning flash. Thus WWLLN stroke detection is effectively also flash detection.

\section{Cross-validation campaign}

We used data recorded during almost the entire 2004 Florida summer thunderstorm season, including all days from 27 April through 30 September. Both LASA and WWLLN archives were parsed down to those events that occurred within $400 \mathrm{~km}$ of $29^{\circ} \mathrm{N}, 82^{\circ} \mathrm{W}$. Table 1 lists the numbers of events from WWLLN and LASA in this study zone, both correlated and uncorrelated.

During the check-out and validation of the LASA 
TABLE 1 . Events during the summer 2004 campaign in the $400-\mathrm{km}$-radius circle centered on $29^{\circ} \mathrm{N}, 82^{\circ} \mathrm{W}$.

No. of WWLLN events

No. of LASA events

No. of WWLLN events with LASA coincidence within $\pm 1 \mathrm{~ms}$

Percent of WWLLN events with LASA coincidence within $\pm 1 \mathrm{~ms}$

No. of LASA events of type CG

No. of LASA events of type IC

No. of WWLLN events with LASA coincidence within $\pm 1 \mathrm{~ms}$ of type $\mathrm{CG}$

No. of WWLLN events with LASA coincidence within $\pm 1 \mathrm{~ms}$ of type IC

* The two entries marked with asterisk sum to 74165 , indicating that $(74165-71362)=2803$ WWLLN events had both CG and IC LASA events within $\pm 1 \mathrm{~ms}$.

system, NLDN data were used as a ground truth against which to evaluate LASA location accuracy (Smith et al. 2002). In a similar spirit, we are now using LASA as a ground truth against which to evaluate WWLLN performance.

Florida is an active lightning region during summer. Its lightning activity tends to occur in airmass thunderstorms that are often regulated by sea-breeze convergence over the Florida Peninsula. The region is particularly poor in positive CG lightning (Carey et al. 2003), so that some aspects of lightning simply cannot be efficiently studied there. Nonetheless, the Florida region is important for characterizing the WWLLN performance statistically, for two reasons. First, Florida is far enough from all circa 2004 WWLLN stations that the signal propagation was necessarily in the earthionosphere waveguide. This is in common with the vast majority of the earth surface, given that WWLLN has extremely sparse station locations. Florida allows us to assess location accuracy in the most relevant regime of WWLLN signal propagation. Second, the study region (a $400-\mathrm{km}$-radius circle centered on $29^{\circ} \mathrm{N}, 82^{\circ} \mathrm{W}$ ) is extremely well covered by LASA and comprises both land and sea surface regions. LASA detects most ( $>80 \%$ of) discharges within this circle if the verticalcurrent-magnitude exceeds several kiloamps, and locates the discharges to within a spatial error on the order of $1 \mathrm{~km}$ (Smith et al. 2002).

LASA detects IC discharges as easily as it detects CG discharges, for comparable currents (Smith et al. 2002). If ICs could be detected at some reasonable efficiency by a VLF system, the system would be provided with at least some ability to monitor certain powerful thunderstorms that have been observed to produce only IC, or mainly IC, activity, and which have been observed not to generate significant CG activity (Lang et al. 2000).
Thus the Florida LASA array provides for some ground truthing of WWLLN's ability (or lack thereof) to locate IC activity.

\section{WWLLN-LASA time coincidences}

We follow well-established practice and measure the distribution of time differences between lightning strokes as measured by LASA and WWLL, conditioned on their respective estimated positions' being within $100 \mathrm{~km}$ of each other. Figure $2 \mathrm{a}$ shows the distribution of LASA-WWLL time differences for coincident events. The top panel is for coincidence to all LASA events, the middle is for coincidence to just CG LASA events, and the bottom is for coincidence to just IC LASA events. The distributions' shoulders (at $\sim \pm 50 \mathrm{~ms}$ ) are from other LASA strokes within the same flash. The right shoulder's greater magnitude, relative to the left shoulder, is due to WWLLN's tendency to record the first stroke of a flash at a higher efficiency than later ones. On the other hand, the closein feature in the middle panel, at $\sim-10 \mathrm{~ms}$, is due to LASA-located leader steps prior to -CGs seen by WWLLN. Noted in parentheses in each panel of Fig. 2a is the number of WWLLN events that have a close $( \pm 1$ ms) LASA coincidence of the respective type; see also Table 1.

There are 75884 WWLLN events in the study having time-of-arrival residuals $<30 \mu$ s and at least five participating stations (see Table 1). Of these, 71362 , or $\sim 94 \%$, have close (within $\pm 1 \mathrm{~ms}$ ) LASA coincidence. That would imply that $\sim 6 \%$ of the WWLLN events (a) may be mislocated (by an error $>100 \mathrm{~km}$ ), (b) may be formed by a misassociation of station triggers, or (c) may actually be detecting valid events that LASA is missing. We have checked this by graphing locations of the WWLLN events that are not accompanied by coincident LASA events, on a map on which locations of the WWLLN events that are accompanied by coincident LASA events are also graphed, for several example 3-h epochs. We find that both classes of WWLLN events are clustered at the same storm centers. We find that 74888 WWLLN events are coincident with LASA in a broader time window of $200 \mathrm{~ms}$. This leaves only 996 (=75 884 - 74 888) WWLLN events that are without even a 200-ms LASA coincidence, that is, only $1.7 \%$ of the WWLLN events. Therefore, we conclude that most of the $6 \%$ of WWLLN events not accompanied by LASA 1-ms coincidences are still valid lightning locations, but of events that were recorded by LASA at the flash level, not at the stroke level.

There are 52728 WWLLN events with close (within $\pm 1 \mathrm{~ms})$ LASA CG coincidence. There are 21437 

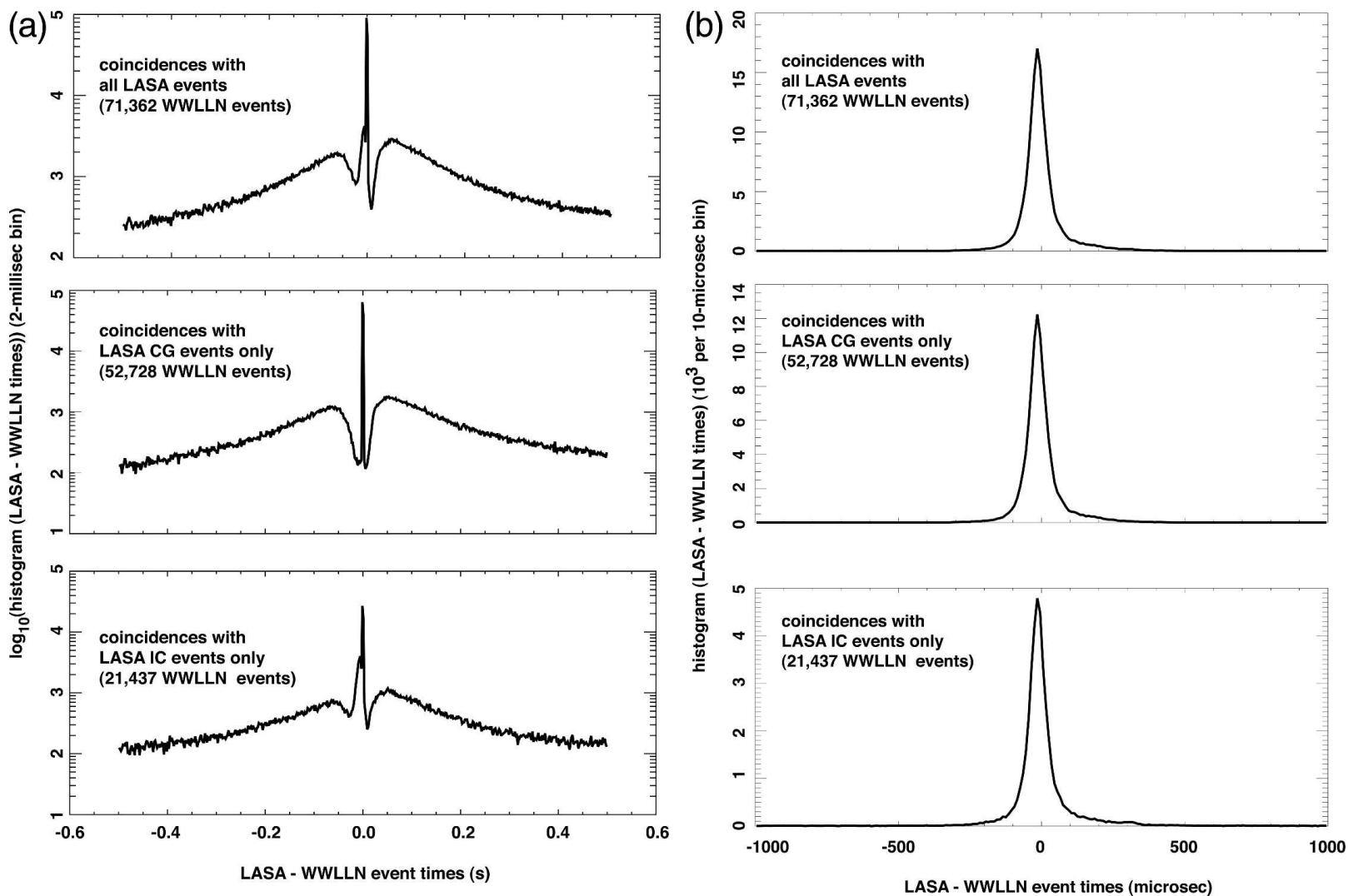

FIG. 2. (a) Distribution of differences between event times estimated by LASA and WWLLN for pairs of events whose estimated positions are within $100 \mathrm{~km}$ of each other. Bin size $=2 \mathrm{~ms}$. In each panel the number in parentheses is the number of WWLLN events having LASA coincidence(s) of the listed type within \pm 1 ms. (top) All LASA events, (middle) LASA CG events, and (bottom) LASA IC events. Note that vetical scale is logarithmic to show sidelobe behavior. (b) As in (a), but for total range of $\pm 1 \mathrm{~ms}$. Bin size $=10$ $\mu \mathrm{s}$. The distributions' half-widths at $1 / e$ are around $50 \mu \mathrm{s}$. Vertical scale is linear, not logarithmic.

WWLLN events with close (within $\pm 1 \mathrm{~ms}$ ) LASA IC coincidence. These last two numbers exceed the overall number of LASA-coincident WWLLN events (71 362) by 2803 , due in part to LASA recording of late leader steps (classified as "IC") within $1 \mathrm{~ms}$ of the stroke, and in part to cloud-level disturbances closely accompanying ground strokes (Jacobson et al. 2000; Suszcynsky et al. 2000, 2001). If we subtract the 2803 WWLLN events that apparently have both CG and IC LASA coincidence, then we still have $21437-2803=18634$ WWLLN events that are due uniquely to IC discharges. This is $\sim 26 \%$ of the WWLLN total events, implying that WWLLN is likely to be able to detect storms that are dominantly IC (Lang et al. 2000), as long as there are NBEs, which alone amongst IC processes tend to produce current amplitudes exceeding $30 \mathrm{kA}$.

Similar to Fig. 2a, Fig. 2b shows the distribution of time differences LASA-WWLL for coincident events, but for the subrange $\pm 1 \mathrm{~ms}$ and with a narrower bin width $(10 \mu \mathrm{s})$. Figure $2 \mathrm{~b}$ shows that the $1 / e$ widths of the time-difference distributions are all on the order of $50 \mu \mathrm{s}$.

\section{LASA-WWLN spatial coincidences}

Figure 3 shows the distributions of eastward (solid curve) and northward (dashed curve) position differences (LASA-WWLLN). WWLLN solutions in Florida have small ( $\sim$ couple of kilometers) westward and northward biases. Since WWLLN uses distant stations at diverse azimuths having diverse ionosphericwaveguide conditions, it is not surprising that a coupleof-kilometers bias is introduced relative to LASA location based on purely ground-wave propagation. This bias apparently is not sufficiently large to spoil WWLLN's association of a lightning discharge with the appropriate convective cell. The $1 / e$ width is in the range $15-20 \mathrm{~km}$. This is small enough to ensure that when WWLLN locates an event, the event is likely to be associated with the appropriate convective cell in the 


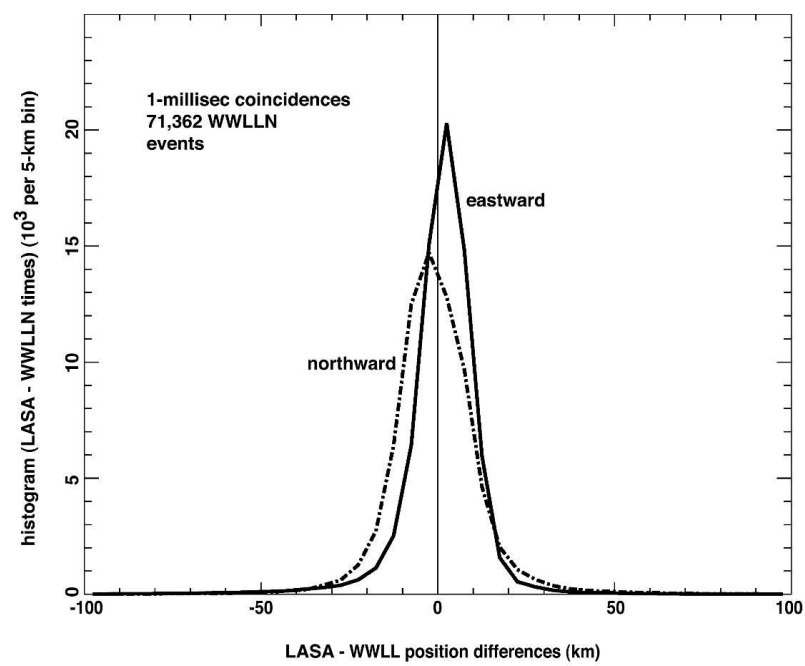

FIG. 3. Distributions of LASA-WWLLN eastward (solid) and northward (dashed) position differences, with bin size $=5 \mathrm{~km}$. Apparently, WWLLN solutions in Florida are biased westward by a couple of kilometers, with a slightly smaller northward bias.

overall storm, but is not small enough to indicate meaningfully the placement of the lightning event within that cell.

\section{WWLLN stroke-detection efficiency}

Figure 4 shows the distributions of LASA-estimated peak vertical current for (a) all LASA events, (b) LASA CG events, and (c) LASA IC events. The dashed curves describe the background LASA population, while the solid curves are for the subset of LASA events having close (within $\pm 1 \mathrm{~ms}$ ) coincidence with WWLLN events. Figure 4 shows several interesting tendencies. First, the background CG distribution (Fig. $4 \mathrm{~b}$, dashed curve) is heavily weighted toward negative CGs, consistent with the negative-CG-dominated climatology of Florida lightning (Carey et al. 2003). Indeed, for practical purposes, we can approximate that there is almost no +CG activity in this study, or at least not enough + CG activity to calibrate the WWLLN detection efficiency for + CG strokes. Second, the IC currents (Fig. 4c, dashed curve) are generally lower than the CG currents, but still extend to several tens of kiloamps. Third, the WWLLN-coincident subset of LASA events systematically occurs at higher current amplitude than does the background distribution of LASA events.

We can estimate a stroke-detection efficiency (stroke DE) for WWLLN by dividing the number of WWLLNcoincident LASA events by the number total background LASA events, separately in each current bin.

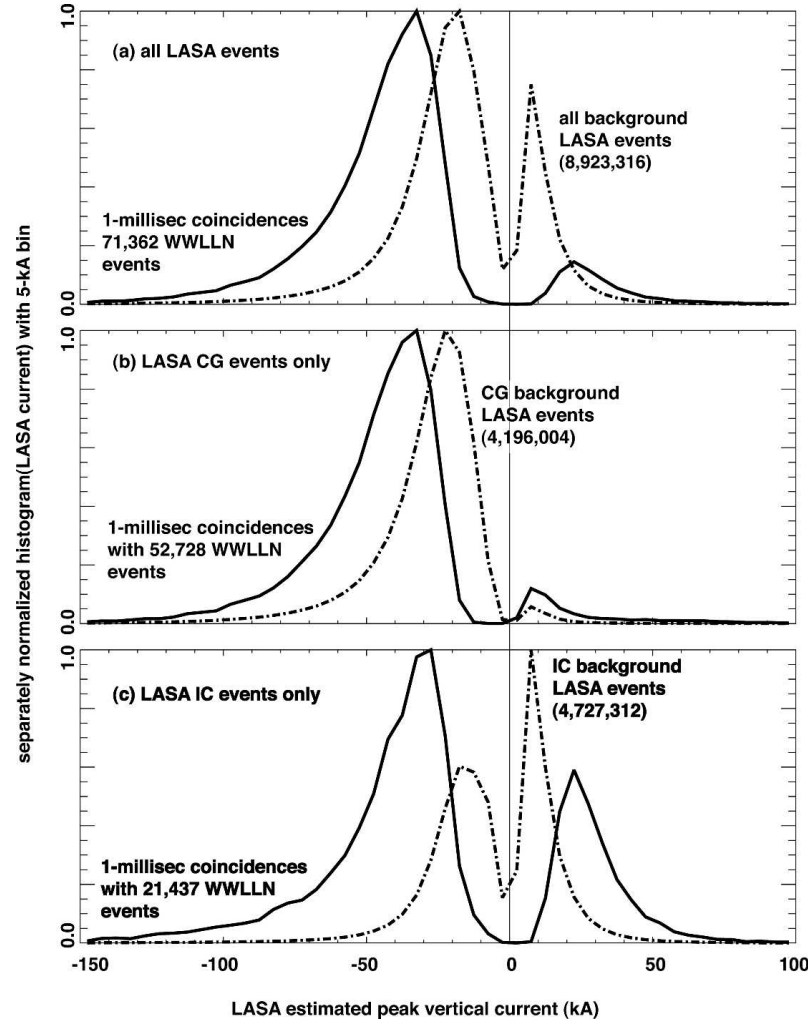

FIG. 4. Distributions of LASA-estimated peak vertical current, with bin size $=5 \mathrm{kA}$. The dashed curve is for the LASA background distribution for (a) all LASA events, (b) LASA CG events, and (c) LASA IC events. The solid curves are for the LASA events (in each type category) having close $( \pm 1 \mathrm{~ms})$ WWLLN coincidence.

The results are shown in Fig. 5. We make two inferences. First, the WWLLN DE, at least for -CGs and ICs, is negligible for current magnitudes $<20 \mathrm{kA}$. Second, the WWLLN DE approaches a few percent for higher (>30 kA) current magnitudes. Apparently, an IC discharge and a -CG discharge are equally detectable for a comparable current. The only reason that more than two-thirds of WWLLN events are nonetheless CG is that (see Fig. 4 above) the current magnitudes of ICs are less than for CGs. Moreover, almost the only ICs with sufficient current to be WWLLNdetectable are NBEs, which have the highest currents of any IC processes (Smith et al. 1999, 2002).

Figure 6 shows similar information as Fig. 5a, but divided into 12-h epochs centered on local (at the LASA centroid) midnight (dashed curve) and local noon (solid curve). The gross changes between midnight- and noon-centered epochs are due to the lossier daytime ionospheric D-region and its effect on longrange VLF propagation of the lightning signal to WWLLN stations. 


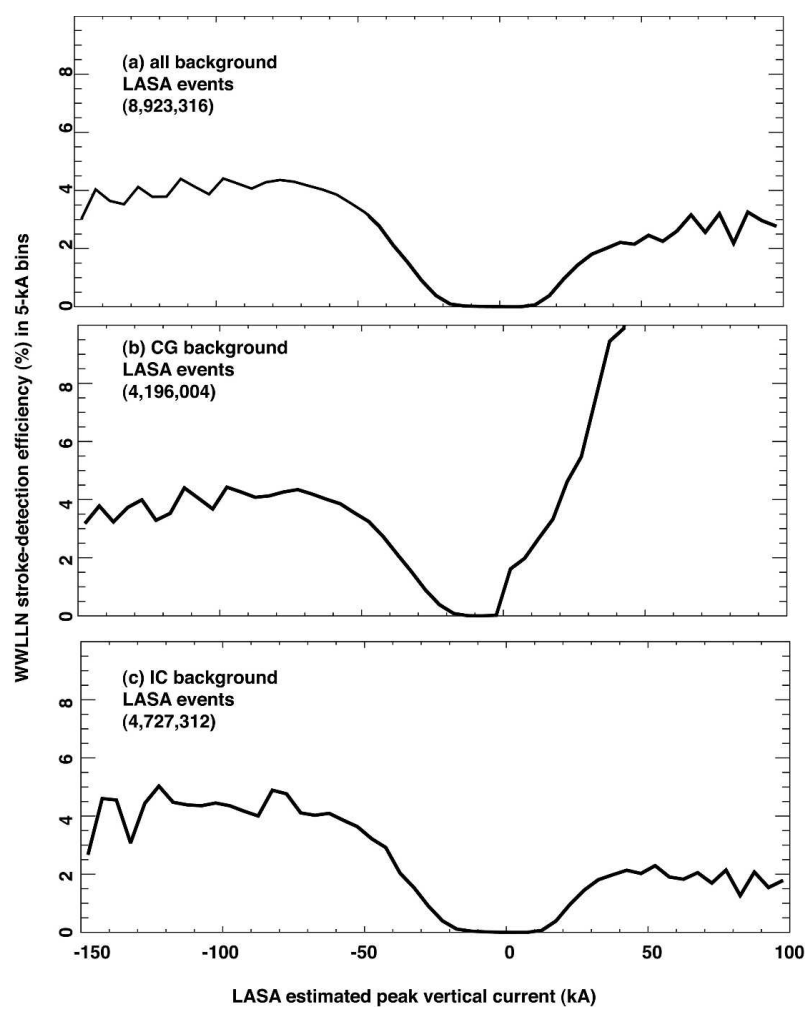

FIG. 5. WWLLN stroke-detection efficiency (stroke DE) as a function of current, with bin size $=5 \mathrm{kA}$. The DE is derived from the ratio of WWLLN-coincident to all LASA background events in each 5-kA-wide bin. (a) All LASA events, (b) LASA CGs, and (c) LASA ICs. The higher apparent DE for positive CGs in (b) is not statistically significant, because it occurs in the near-absence of background LASA events (see Fig. $4 \mathrm{~b}$ above). The legend in (a)-(c) lists the number of LASA background events for that stroke type.

\section{WWLLN storm-detection efficiency}

It is clear that WWLLN and similar VLF long-range lightning-detection systems will never provide the focused, detailed view of the interior of a thunderstorm that is provided by various radio-frequency lightning mappers such as LMA. Indeed, VLF systems will never offer the level of detail or detection efficiency even of satellite-based optical imagers. Instead, the products which long-range VLF systems might be able to provide are (a) synoptic location of current convective cells and (b) statistical inference of the rain rate (and hence latent-heat-release rate) to the extent that empirical rain/ lightning parameterizations have any skill within the particular meteorological setting. Both of these observational products must be available promptly (though not strictly in real time). Here, "promptly" means within, say, $\sim 3 \mathrm{~h}$, so as to permit data assimilation into model forecast update cycles. Additionally, both of

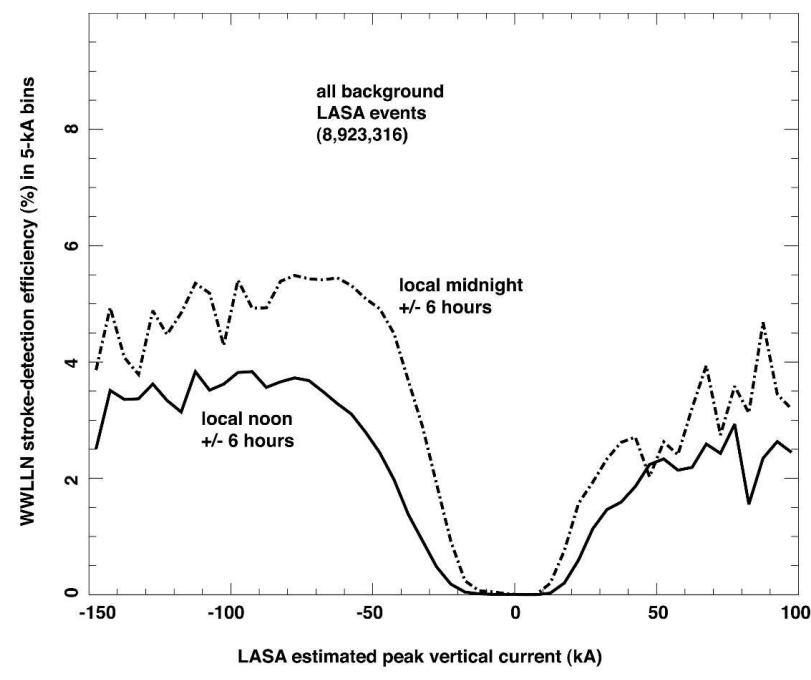

FIG. 6. Similar to Fig. 5a, but divided into 12-h epochs centered on local (at the LASA centroid) midnight (dashed curve) and noon (solid curve). The gross changes are due to the lossier daytime ionospheric propagation of the lightning VLF signal to WWLLN stations.

these products must localize a storm spatially to within a reasonable scale of mesoscale-forecast variation, for example, $20 \mathrm{~km}$.

To provide product (a), a VLF system must not miss significant storms within their 3-h evolution. Similary, a VLF system must not falsely detect storms that do not in fact exist. To provide product (b), a VLF system must, even though its stroke DE is low, have a stroke DE that is statistically, but not strictly, proportional within a given meteorological regime (e.g., continental, maritime, island, etc.). If the DE were not statistically proportional, but instead varied randomly from storm to storm even in a fixed meteorological setting, then the detected lightning rate would provide no skill as a proxy for rainfall. If the DE were strictly proportional, then we could calibrate it. WWLLN seems to be better than unproportional, and worse than strictly proportional. An exemplary case (Chang et al. 2001) of lightning data assimilation where these requirements are met showed significant improvements in mesoscaleforecast skill. Similarly, recently it has been found that VLF long-range detection of lightning in remote, noncoastal Pacific maritime storms can be of use when assimilated into forecasts in that poorly covered region (Pessi et al. 2004). Both of these references provide background material on the utility, and also the limitations, of treating lightning stroke rates as a proxy for rainfall.

We will now test the ability of WWLLN to detect the same storms as detected by LASA, and to detect strokes at a rate that is statistically proportional to the 
(a) all LASA (max $=700)$

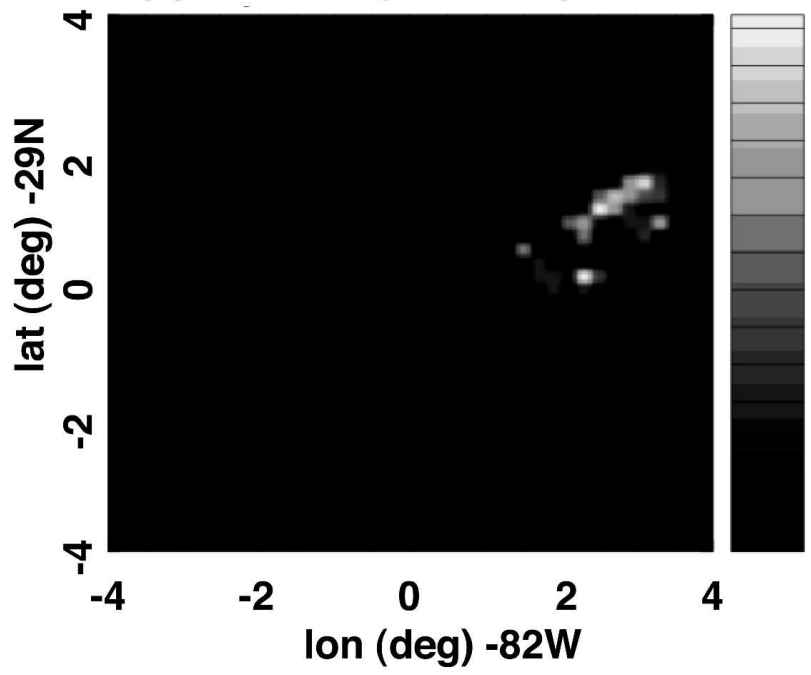

(c) LASA CGs $(\max =550)$

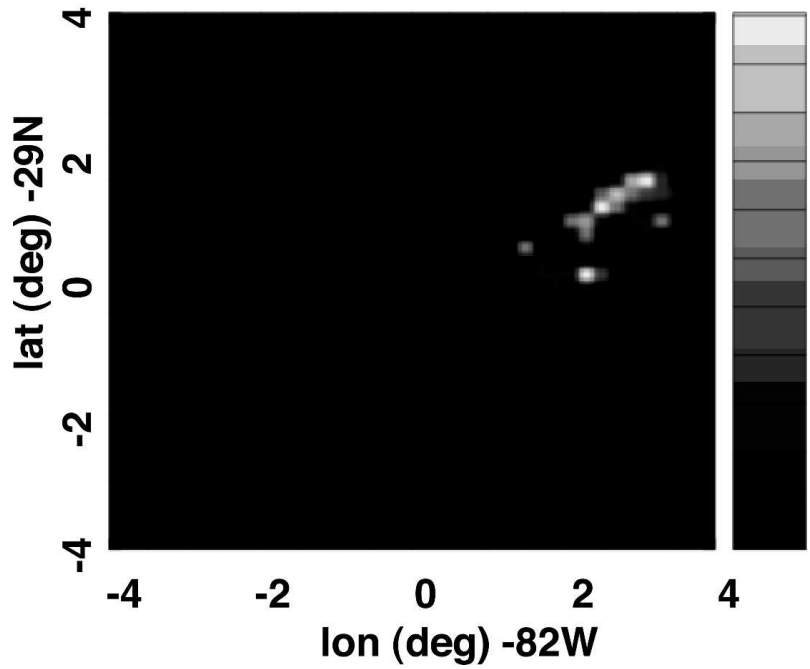

(b) all WWLLN $(\max =90)$

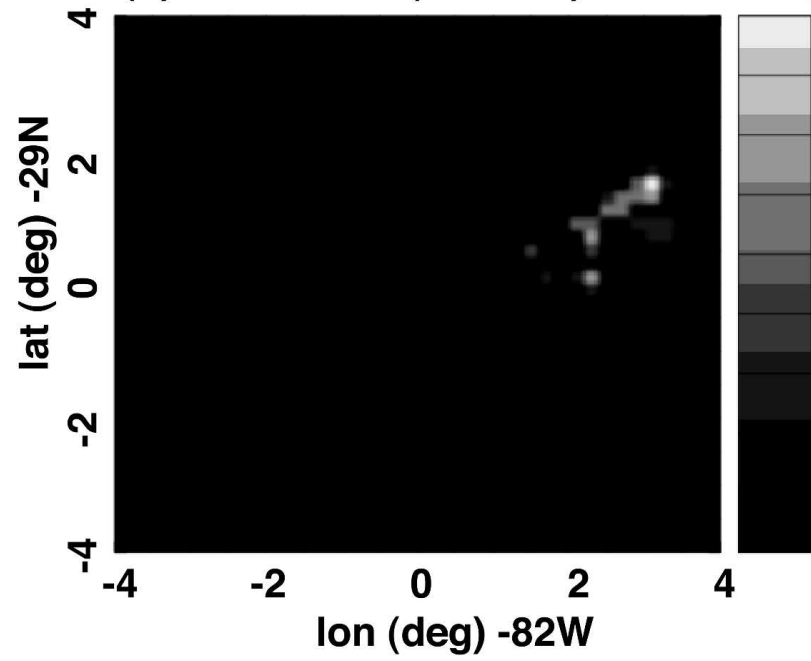

(d) LASA ICs ( $\max =65)$

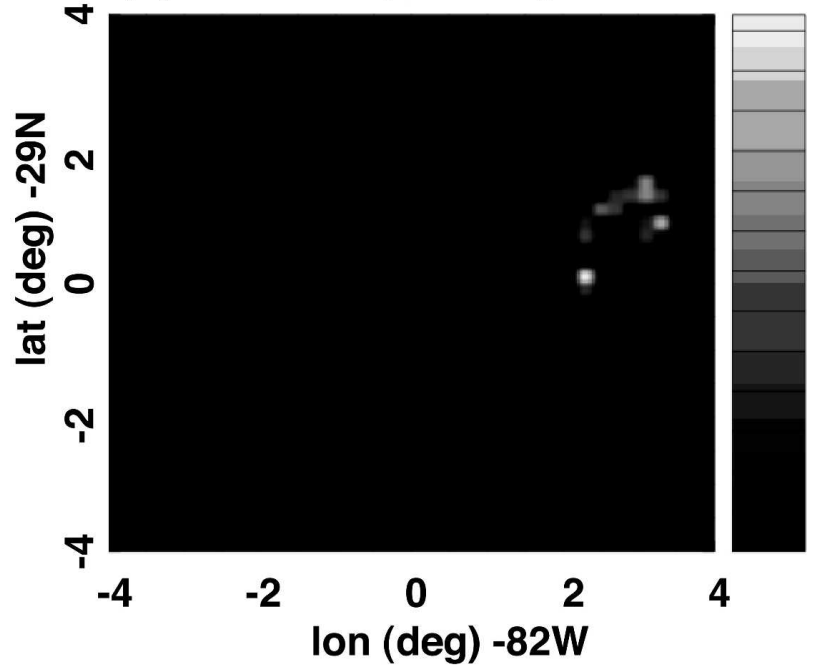

FIG. 7. Pixel maps of lightning-event occurrence during the 3 -h period (0600-0900 UTC) on 13 Aug 2004 . The map center is at $29^{\circ}$ N, $82^{\circ} \mathrm{W}$ (the array center), and each axis extends $\pm 4^{\circ}$ about the center. Pixel size is $0.2^{\circ} \times 0.2^{\circ}$; there are 1600 spatial pixels. (a) All LASA events, (b) all WWLLN events, (c) LASA CG events, and (d) LASA IC events. Each legend in (a)-(d) lists the max value of pixel occupancy, corresponding to the lightest shade at the top of the grayscale. In all boxes, the bottom of the grayscale (black) corresponds to zero occupancy.

strokes detected by LASA. We divide each day of our campaign into eight periods, each of 3-h duration, for the entire campaign from 27 April through 30 September. Next, the WWLLN and LASA lightning events are each put into a matrix of locations. The matrix pixel size is $0.2^{\circ} \times 0.2^{\circ}$ in both latitude and longitude. There are $40 \times 40$ pixels, so that the covered field ranges over $\pm 4^{\circ}$ in both latitude and longitude about the array center $\left(29^{\circ} \mathrm{N}, 82^{\circ} \mathrm{W}\right)$. Figure 7 shows an example of lightningoccurrence pixel maps during a single 3 -h period on 13 August 2004. The various pixel-occupancy matrices are computed and stored for all 3-h epochs in the campaign.
Let $\mathbf{N}_{L}(j, k, m)$ be the $40 \times 40$ matrix for all LASA events, and let $\mathbf{N}_{W}(j, k, m)$ be the $40 \times 40$ matrix for all WWLLN events in 3-h epoch $m$.

During each 3-h period, we tally three statistics:

1) the sum of the square of WWLLN pixel occupancy (proportional to autovariance):

$$
\mathbf{A}_{W m}=\sum_{j=1,40} \sum_{k=1,40} N_{W}^{2}(j, k, m)
$$

2) the sum of the square of LASA pixel occupancy (proportional to autovariance): 
(a)

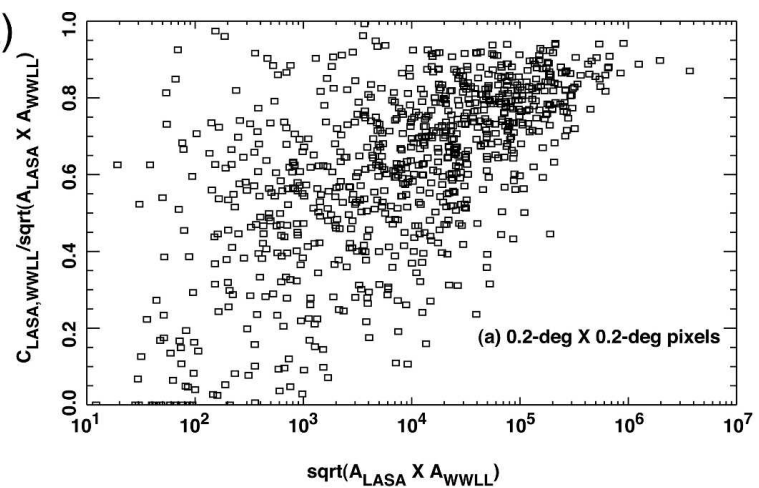

(b)

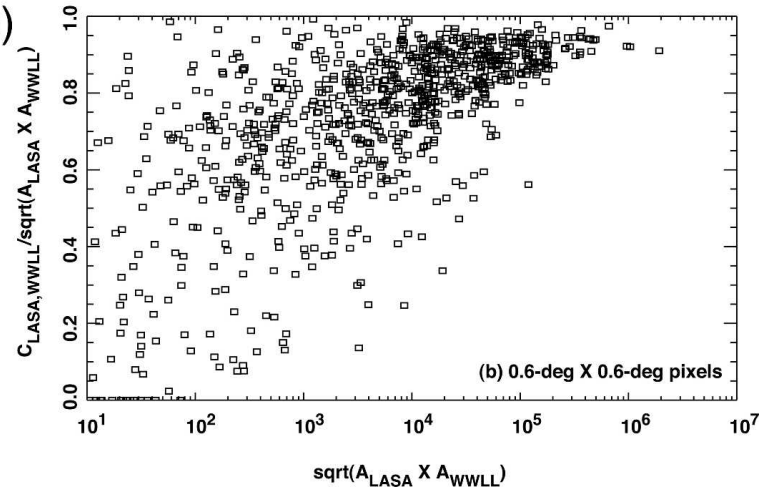

FIG. 8. Scatterplot of normalized correlation (vertical axis) of LASA and WWLLN pixel occupancies vs geometric mean of LASA and WWLLN pixel-occupancy autovariances. Each data point is for a different 3-h epoch during the summer 2004 campaign. (a) Unsmoothed, using the original $0.2^{\circ} \times 0.2^{\circ}$ pixels; (b) smoothed, using $0.6^{\circ} \times 0.6^{\circ}$ pixels.

$$
\mathbf{A}_{L m}=\sum_{j=1,40} \sum_{k=1,40} N_{L}^{2}(j, k, m)
$$

3) the sum of product of WWLLN and LASA pixeloccupancy matrices (proportional to equal-time covariance):

$$
\mathbf{C}_{L W m}=\sum_{j=1,40} \sum_{k=1,40} \mathbf{N}_{L}(j, k, m) \times \mathbf{N}_{W}(j, k, m)
$$

The normalized spatial correlation between WWLLN and LASA is the ratio of $\mathbf{C}_{L W m}$ to the geometric mean of $\mathbf{A}_{L m}$ and $\mathbf{A}_{W m}$. Figure 8 is a scatterplot of the normalized correlation (vertical axis) versus the geometric mean of the WWLLN and LASA autovariances $\left(\mathbf{A}_{L m}\right.$ and $\mathbf{A}_{W m}$, respectively; horizontal axis). Each point is a separate 3 -h epoch. Figure $8 \mathrm{a}$ is for the native $0.2^{\circ} \times$ $0.2^{\circ}$ pixels. Figure $8 \mathrm{~b}$ is for $3 \times 3$ smoothing of the original $0.2^{\circ} \times 0.2^{\circ}$ pixels, that is, effectively for $0.6^{\circ} \times$ $0.6^{\circ}$ pixels. For the upper two orders of magnitude on the horizontal axis, most of the epochs' correlations are around $80 \%$ or higher, except for Poisson noise in the lower-left (low-lightning-rate) part of the relationship.
The larger-pixel case (Fig. 8b) has higher correlations than the smaller-pixel case (Fig. 8a).

Figure 8 indicates that WWLLN (despite its relatively low stroke-detection efficiency) detects storms with a high degree of spatial fidelity within a given 3-h epoch, based on the use of LASA as a benchmark. This is done despite the Florida stroke-detection rate for WWLLN being less than $1 \%$ overall of that for LASA (see Table 1). The evidence of Fig. 8 implies that WWLLN can provide useful lightning-location data for assimilation in model forecasts on a 3-h time scale.

The evidence of Fig. 8 addresses spatial fidelity of WWLLN data, compared to LASA. However, the question is not yet answered as to whether the WWLLN event counts are proportional, in a consistent way (over the many 3-h epochs), to the LASA event counts. We can make this argument about proportionality by considering not just proportionality within $3-\mathrm{h}$ epochs (as done above in Fig. 8), but also overall proportionality over the entire 2004 cross-validation campaign. We take the entire campaign-summed total of each of the quantities defined in Eqs. (1)-(3), and then evaluate the campaign-global correlation

$$
\mathbf{C}_{\text {global }}=\sum_{m} \mathbf{C}_{L W_{m}} / \sqrt{\left(\sum_{m} \mathbf{A}_{L_{m}}\right) \times\left(\sum_{m} \mathbf{A}_{W_{m}}\right)} .
$$

We find that the global correlation so defined is $60.5 \%$, indicating that WWLL over the course of an entire summer detects storms with stroke detections that are partially proportional to the stroke detections per storm by LASA. We find that a major part of the correlation's departure from $100 \%$ is due to the averaging over all local times. To show this, we have broken this exercise into eight universal coordinated time bins, each $3 \mathrm{~h}$ long. Summing over all days in this study, the correlations for the eight 3 -h periods are $65.6 \%$ (0000-0300 UTC), 68.9\% (0300-0600 UTC), 75.4\% (0600-0900 UTC), 62.6\% (0900-1200 UTC), 72.6\% (1200-1500 UTC), 67.8\% (1500-1800 UTC), 68.7\% (1800-2100 UTC), and 73.8\% (2100-2400 UTC). Each of these time slices has a higher WWLLN/LASA internal proportionality than does the overall database $(60.5 \%)$. This means that the efficiency of WWLLN in the Florida area varies with universal coordinated time. Further work on this effect will be required before we can reduce this form of variability in WWLLN "calibration" in its estimates of lightning stroke rate.

\section{Conclusions}

The overall stroke-detection efficiency for WWLLN is less than $1 \%$. However, strokes at higher current 
amplitudes ( $>30 \mathrm{kA})$ are detected at $\sim 4 \%$ efficiency. This is independent of the type and polarity of the stroke, and apparently depends only on stroke-current amplitude.

About half of the strokes LASA detects in Florida are ICs. WWLLN stroke detections in Florida are 26\% IC. This indicates that WWLLN is able to detect storms that are dominantly IC, provided that some of the IC discharges are sufficiently high current.

WWLLN stroke detections, when they occur, are accurate to within $50 \mu \mathrm{s}$ in time and $15-20 \mathrm{~km}$ in position. This spatial accuracy permits identification of the convective cell but not a finer-resolution analysis of the discharge position within the storm complex.

Despite its low overall stroke DE, and the steep selectivity of that DE for high-current amplitudes, WWLLN supplies a spatially accurate and representative census of storms on a 3-h averaging epoch. Moreover, the number of WWLLN strokes in these storms appears to be statistically proportional to the number of LASA strokes, though with as yet unmodeled localtime variations. When these variations are finally understood and modeled, then we expect that WWLLN will be able to furnish 3-h-update data assimilation (e.g., of latent-heat-release rate, or rain rate) into model forecasts, to the extent that one may prudently use lighting rate as a proxy for rain rate.

Acknowledgments. We are indebted to the entire LASA team for their kind cooperation. Two authors (ARJ and JH) were supported by the U.S. Department of Energy, under whose auspices the LASA array is operated. Two authors (RHH and EHL) were supported in part by a grant from the Mindlin Foundation. WWLLN data are provided through the University of Washington under agreement with Low Frequency Electromagnetics of New Zealand. ARJ was a visiting scholar at the University of Washington. We have profited from insightful suggestions by Craig Rodger.

\section{REFERENCES}

Boccippio, D. J., W. J. Koshak, H. J. Christian, and S. J. Goodman, 1999: Land-ocean differences in LIS and OTD tropical lightning observations. Proc. 11th Int. Conf. on Atmospheric Electricity, Huntsville, AL, National Aeronautics and Space Administration, 734-737.

-, K. Driscoll, W. Koshak, R. Blakeslee, W. Boeck, D. Buechler, H. Christian, and S. Goodman, 2000a: The Optical Transient Detector (OTD): Instrument characteristics and cross-sensor validation. J. Atmos. Oceanic Technol., 17, 441458.

— S. J. Goodman, and S. Heckman, 2000b: Regional differences in tropical lightning distributions. J. Appl. Meteor., 39, 2231-2248.
Carey, L. D., S. A. Rutledge, and W. A. Petersen, 2003: The relationship between severe storm reports and cloud-to-ground lightning polarity in the contiguous United States from 1989 to 1998. Mon. Wea. Rev., 131, 1211-1228.

Chang, D.-E., J. A. Weinman, C. A. Morales, and W. S. Olson, 2001: The effect of spaceborne microwave and ground-based continuous lightning measurements on forecasts of the 1998 Groundhog Day Storm. Mon. Wea. Rev., 129, 1809-1833.

Christian, H. J., R. J. Blakeslee, and S. J. Goodman, 1989: The detection of lightning from geostationary orbit. J. Geophys. Res., 94, 13 329-13 337.

, and Coauthors, 1999a: Global frequency and distribution of lightning as observed by the Optical Transient Detector (OTD). Proc. 11th Int. Conf. on Atmospheric Electricity, Huntsville, AL, National Aeronautics and Space Administration, 726-729.

— 11th Int. Conf. on Atmospheric Electricity, Huntsville, AL, National Aeronautics and Space Administration, 746-749.

Cummins, K. L., M. J. Murphy, E. A. Bardo, W. L. Hiscox, R. Pyle, and A. E. Pifer, 1998: Combined TOA/MDF technology upgrade of U.S. National Lightning Detection Network. J. Geophys. Res., 103, 9035-9044.

Dowden, R. L., J. B. Brundell, and C. J. Rodger, 2002: VLF lightning location by time of group arrival (TOGA) at multiple sites. J. Atmos. Sol.-Terr. Phys., 64, 817-830.

Jacobson, A. R., and M. J. Heavner, 2005: Comparison of narrow bipolar events with ordinary lightning as proxies for severe convection. Mon. Wea. Rev., 133, 1144-1154.

_, K. L. Cummins, M. Carter, P. Klingner, D. Roussel-Dupré, and S. O. Knox, 2000: FORTE radio-frequency observations of lightning strokes detected by the National Lightning Detection Network. J. Geophys. Res., 105, 15 653-15 662.

Lang, T. J., S. A. Rutledge, J. E. Dye, M. Venticinque, P. Laroche, and E. Defer, 2000: Anomalously low negative cloud-toground lightning flash rates in intense convective storms observed during STERAO-A. Mon. Wea. Rev., 128, 160-173.

Lay, E. H., R. H. Holzworth, C. J. Rodger, J. N. Thomas, O. Pinto, and R. L. Dowden, 2004: WWLL global lightning detection system: Regional validation study in Brazil. Geophys. Res. Lett., 31, L03102, doi:10.1029/2003GL018882.

Lee, A. C. L., 1986a: An operational system for the remote location of lightning flashes using a VLF arrival time difference technique. J. Atmos. Oceanic Technol., 3, 630-642.

_ 1986b: An experimental study of the remote location of lightning flashes using a VLF arrival time difference technique. Quart. J. Roy. Meteor. Soc., 112, 203-229.

Lyons, W. A., T. E. Nelson, E. R. Williams, J. A. Cramer, and T. R. Turner, 1998: Enhanced positive cloud-to-ground lightning in thunderstorms ingesting smoke from fires. Science, 282, 77-80.

Orville, R. E., 1994: Cloud-to-ground lightning flash characteristics in the contiguous United States. J. Geophys. Res., 99, $10833-10841$.

—_, and Coauthors, 2001: Enhancement of cloud-to-ground lightning over Houston, Texas. Geophys. Res. Lett., 28, $2597-$ 2600.

Pessi, A., S. Businger, K. L. Cummins, and T. Turner, 2004: On the relationship between lightning and convective rainfall over the central Pacific Ocean. Proc. 18th Int. Lightning Detection Conf., Helsinki, Finland, Vaisala, CD-ROM, 21.

Rison, W., R. J. Thomas, P. R. Krehbiel, T. Hamlin, and J. Harlin, 1999: A GPS-based three-dimensional lightning mapping sys- 
tem: Initial observations in central New Mexico. Geophys. Res. Lett., 26, 3573-3576.

Rodger, C. J., J. B. Brundell, R. L. Dowden, and N. R. Thomson, 2004: Location accuracy of long distance VLF lightning location network. Ann. Geophys., 22, 747-758.

- _ — and - 2005: Location accuracy of VLF World Wide Lightning Location (WWLL) network: Post-algorithm upgrade. Ann. Geophys., 23, 277-290.

Shao, X.-M., M. Stanley, A. Regan, J. Harlin, M. Pongratz, and M. Stock, 2006: Total lightning observations with the new and improved Los Alamos sferic array (LASA). J. Atmos. Oceanic Technol., in press.

Smith, D. A., and Coauthors, 1999: A distinct class of isolated intracloud lightning discharges and their associated radio emissions. J. Geophys. Res., 104, 4189-4212.

, K. B. Eack, J. Harlin, M. J. Heavner, A. R. Jacobson, R. S. Massey, X. M. Shao, and K. C. Wiens, 2002: The Los Alamos sferic array: A research tool for lightning investigations. $J$. Geophys. Res., 107, 4183, doi:10.1029/2001JD000502.

— M. J. Heavner, A. R. Jacobson, X. M. Shao, R. S. Massey, R. J. Sheldon, and K. C. Wiens, 2004: A method for determining intracloud lightning and ionospheric heights from
VLF/LF electric field records. Radio Sci., 39, RS1010, doi:10.1029/2002RS002790.

Stanley, M. A., M. Pongratz, X. Shao, J. Harlin, and A. Regan, 2004: The new LANL sferic array: Operation and calibration. Eos, Trans. Amer. Geophys. Union, 85, F266.

Suszcynsky, D. M., M. W. Kirkland, A. R. Jacobson, R. C. Franz, S. O. Knox, J. L. L. Guillen, and J. L. Green, 2000: FORTE observations of simultaneous VHF and optical emissions from lightning: Basic phenomenology. J. Geophys. Res., 105, 2191-2201.

_, T. E. Light, S. Davis, M. W. Kirkland, J. L. Green, and J. Guillen, 2001: Coordinated observations of optical lightning from space using the FORTE photodiode detector and CCD imager. J. Geophys. Res., 106, 17 897-17 906.

Thomas, R. J., P. R. Krehbiel, W. Rison, T. Hamlin, D. J. Boccippio, S. J. Goodman, and H. J. Christian, 2000: Comparison of ground-based 3-dimensional lightning mapping observations with satellite-based LIS observations in Oklahoma. Geophys. Res. Lett., 27, 1703-1706.

,,,---- J. Harlin, and D. Shown, 2001: Observations of VHF source powers radiated by lightning. Geophys. Res. Lett., 28, 143-146. 